\title{
The Some Properties of Skew Polynomial Rings
}

\author{
Qianqian Chu, Dan Li, Hailan Jin* \\ Department of Mathematics, College of Sciences, Yanbian University, Yanji, China \\ Email: "hljin98@ybu.edu.cn, "hljin98@hanmail.net
}

Received 5 May 2016; accepted 17 June 2016; published 20 June 2016

Copyright (C) 2016 by authors and Scientific Research Publishing Inc.

This work is licensed under the Creative Commons Attribution International License (CC BY). http://creativecommons.org/licenses/by/4.0/

c) (†) Open Access

\section{Abstract}

This paper mainly studies some properties of skew polynomial ring related to Morita invariance, Armendariz and (quasi)-Baer. First, we show that skew polynomial ring has no Morita invariance by the counterexample. Then we prove a necessary condition that skew polynomial ring constitutes Armendariz ring. We lastly investigate that condition of skew polynomial ring is a (quasi)-Baer ring, and verify that the conditions is necessary, but not sufficient by example and counterexample.

\section{Keywords}

Skew Polynomial Ring, (Quasi)-Baer Ring, Armendariz Ring, Morita Context Ring, Morita Invariance, Nozero Divisor Ring

\section{Introduction}

Throughout this paper every ring is an associative with identity unless otherwise stated. Given a ring $R, R[x]$, $R[x ; \sigma], r_{R}(X), l_{R}(X), \operatorname{Mat}_{n}(R)$ and $\mathbb{Z}_{n}$ denote the polynomial ring with an indeterminate $x$ over $R$, the skew polynomial ring over $R$, the right annihilator of nonempty subset $X$ of ring $R$, the left annihilator of nonempty subset $X$ of ring $R$, and the $n \times n$ matrix ring over $R$, the ring of integers modulo $n$, respectively. A ring $R[x ; \sigma]$ is called Skew polynomial ring if $\sigma$ is an endomorphism over $R$; operations are usual addition and multiplication defined by $x a=\sigma(a) x, a \in R$. In [1], that skew polynomial ring has no Morita invariance. A ring $R$ is called Armendariz ring if $f(x) g(x)=0$ implies $a_{i} b_{j}=0$, where $0 \leq i \leq m, 0 \leq j \leq n$ for any $f(x)=\sum_{i=0}^{m} a_{i} x^{i}, g(x)=\sum_{j=0}^{n} b_{j} x^{j} \in R[x]$ in [2]. If $R$ is a semiprime ring, then skew polynomial ring $R[x ; \sigma]$ is a quasi-Armendariz ring by [3]. G. F. Birkenmeier first introduced the concept of Baer ring, and

\footnotetext{
"Corresponding author.
} 
proved that Baer ring is quasi-Baer ring, but converse is not hold, and right principally quasi-Baer ring has Morita invariance by [4]. Q.J. Song gave the condition that iterated skew polynomial ring constitutes (quasi)-Baer ring by [5]. We will show that skew polynomial ring has no Morita invariance by the counterexample, and the condition that skew polynomial ring has properties of Armendarizand (quasi)-Baer, and verify that the condition is necessary, but not sufficient by example and counterexample.

\section{Preliminary}

Definition 2.1. [6] Let $R$ and $S$ be rings, then $R$ and $S$ are Morita equivalent if there exists projective module $W_{R}$, such that $S \cong$ End $W_{R}$. Morita invariance is the invariant property under Morita equivalent rings.

Lemma 2.2. [6] The ring $R$ and $S$ are Morita equivalent, if and only if there exists an integer $n$ and idempotent $e \in \operatorname{Mat}_{n}(R)$, such that $S \cong e \operatorname{Mat}_{n}(R) e$ and $\operatorname{Mat}_{n}(R) e \operatorname{Mat}_{n}(R)=\operatorname{Mat}_{n}(R)$.

Definition 2.3. [7] A ring $R$ is called (quasi)-Baer ring if the right annihilator of (resp. right ideal) nonempty subset of $R$ is generated by an idempotent as a right ideal.

Lemma 2.4. Suppose that $R$ is a ring has no zero divisor and $\sigma$ is a monomorphism over $R$, then skew polynomial ring $R[x ; \sigma]$ has no zero divisor.

Proof. For any $f(x)=\sum_{i=0}^{m} a_{i} x^{i}, g(x)=\sum_{j=0}^{n} b_{j} x^{j} \in R[x]$, if

$$
\begin{aligned}
f(x) \cdot g(x)= & \sum_{i+j=0}^{n+m} a_{i} \sigma^{i}\left(b_{j}\right) x^{i+j}=a_{0} b_{0}+\left(a_{1} \sigma\left(b_{0}\right)+a_{0} b_{1}\right) x+\left(a_{0} b_{2}+a_{1} \sigma\left(b_{1}\right)+a_{2} \sigma^{2}\left(b_{0}\right)\right) x^{2} \\
& +\cdots+a_{n} \sigma^{n}\left(b_{m}\right) x^{n+m}=0
\end{aligned}
$$

then all coefficients of the skew polynomial are zero. Since $\sigma$ is a monomorphism and $R$ has no zero divisor, so $\sigma^{i}\left(b_{j}\right)=0$ implies $b_{j}=0, a_{0} b_{0}=0$ implies $a_{0}=0$ or $b_{0}=0$. Case 1 . If $a_{0}=0, b_{0} \neq 0$, then $\sigma^{i}\left(b_{0}\right) \neq 0$. Since $a_{1} \sigma\left(b_{0}\right)+a_{0} b_{1}=0$, so $a_{1}=0$. Simlarly, we show $a_{i}=0 \quad(0 \leq i \leq n)$, thus $f(x)=0$. Case 2. If $b_{0}=0, a_{0} \neq 0$, then $\sigma^{i}\left(b_{0}\right)=0$. Because $a_{1} \sigma\left(b_{0}\right)+a_{0} b_{1}=0$, so $b_{1}=0, \sigma^{i}\left(b_{1}\right)=0$. Similarly, $b_{j}=0 \quad(0 \leq j \leq m)$, thus $g(x)=0$. Case 3. If $a_{0}=b_{0}=0, a_{0} b_{2}+a_{1} \sigma\left(b_{1}\right)+a_{2} \sigma^{2}\left(b_{0}\right)=0$, so $\sigma^{i}\left(b_{0}\right)=0$, $a_{1} \sigma\left(b_{1}\right)=0$. Similarly, $a_{i}=0$ or $b_{j}=0$ for $0 \leq i \leq m, 0 \leq j \leq n$, then $f(x)=0$ or $g(x)=0$.Therefore $R[x ; \sigma]$ is a ring has no divisor of zero.

Definition 2.5. [8] A ring $\mathrm{R}$ is called a reversible, if $a b=0$ implies $b a=0$ for any $a, b \in R$.

Proposition 2.6. [9] Every reduced ring is a reversible ring, but the converse does not hold.

Proposition 2.7. Let $R$ be a reduced ring, then the coefficients of right annihilator of any polynomial over $R[x]$ are the right annihilator of all coefficients of the polynomial.

Proof. For any $f(x)=\sum_{i=0}^{n} a_{i} x^{i}, g(x)=\sum_{j=0}^{m} b_{j} x^{j} \in R[x]$, let $f(x) \cdot g(x)=0$, then

$f(x) \cdot g(x)=\left(\sum_{i=0}^{n} a_{i} x^{i}\right)\left(\sum_{j=0}^{m} b_{j} x^{j}\right)=\sum_{i+j=0}^{n+m} a_{i} \sigma^{i}\left(b_{j}\right) x^{i+j}=0$, so $a_{0} b_{0}=0, a_{0} b_{1}+a_{1} b_{0}=0$,

$a_{0} b_{2}+a_{1} b_{1}+a_{2} b_{0}=0, \cdots, a_{n} b_{n}=0$ and $b_{0} \in r_{R}\left(a_{0}\right)$. Because $R$ be a reduced ring, hence $R$ be a reversible ring, so $b_{0} a_{0}=0$. Because $a_{0} b_{1}+a_{1} b_{0}=0$, then $b_{0} a_{0} b_{1}+b_{0} a_{1} b_{0}=0$, so $b_{0} a_{1} b_{0}=0$, so $a_{1} b_{0}=0$, thus $b_{0} \in r_{R}\left(a_{1}\right)$ and $b_{0} a_{1}=0$, so $a_{0} b_{1}=0$, so $b_{1} \in r_{R}\left(a_{0}\right)$ and $b_{1} a_{0}=0$. Since $a_{0} b_{2}+a_{1} b_{1}+a_{2} b_{0}=0$, we have $a_{0} b_{2} a_{0}+a_{1} b_{1} a_{0}+a_{2} b_{0} a_{0}=0$, then $a_{0} b_{2} a_{0}=0$, so $b_{2} a_{0}=0$, so $b_{2} \in r_{R}\left(a_{0}\right)$ and $a_{0} b_{2}=0$, so $a_{1} b_{1}+a_{2} b_{0}=0$, so $a_{1} b_{1} a_{1}=0$, hence $a_{1} b_{1}=0$, so $b_{1} \in r_{R}\left(a_{1}\right), b_{1} a_{1}=0$, so $a_{2} b_{0}=0$, thus $b_{0} \in r_{R}\left(a_{2}\right)$, Similarly, we have $b_{i} \in r_{R}\left(a_{j}\right)$, which $i=0,1,2, \cdots, n, j=0,1, \cdots, m$. Therefore the coefficients of right annihilator of any polynomial over $R[x]$ are the right annihilator of all coefficients of the polynomial.

Proposition 2.8. Let $\mathrm{R}$ be a reduced ring, then the idempotent of ring $\mathrm{R}$ is the idempotent of $R[x]$.

Proof. For any $f(x)=a_{0}+a_{1} x+\cdots+a_{n} x^{n} \in R[x]$, if $f^{2}(x)=f(x)$, we have

$$
\begin{aligned}
& \left(a_{0}+a_{1} x+a_{2} x^{2}+\cdots+a_{n} x^{n}\right)^{2} \\
= & a_{0}^{2}+\left(a_{0} a_{1}+a_{1} a_{0}\right) x+\left(a_{0} a_{2}+a_{1} a_{1}+a_{2} a_{0}\right) x^{2}+\cdots+\left(a_{0} a_{n}+a_{1} a_{n-1} x+a_{2} a_{n-2} x^{2}+\cdots+a_{n} a_{0}\right) x^{n} \\
& +\left(a_{1} a_{n}+a_{2} a_{n-1}+a_{3} a_{n-2}+\cdots+a_{n} a_{1}\right) x^{n+1}+\cdots+\left(a_{n-1} a_{n}+a_{n} a_{n-1}\right) x^{2 n-1}+\left(a_{n} a_{n}\right) x^{2 n} \\
= & a_{0}+a_{1} x+a_{2} x^{2}+a_{3} x^{3}+\cdots+a_{n} x^{n}
\end{aligned}
$$

then $\sum_{i+j=n+1}^{n+m} a_{i} \sigma^{i}\left(b_{j}\right) x^{i+j}=0$. Since $\mathrm{R}$ is a reduced ring and $a_{n}^{2}=0, a_{n-2} a_{n}+\left(a_{n-1}\right)^{2}+a_{n} a_{n-2}=0$, so $a_{n}=0$, 
$a_{n-1}=0$. Similarly, we have $a_{n-2}=\cdots=a_{2}=0$, and because $a_{0} a_{2}+a_{1}^{2}+a_{2} a_{0}=0$, so $a_{1}=0$. Thus $f(x)=a_{0}$, which $a_{0}^{2}=a_{0} \in R$ is the idempotent of $R[x]$.

\section{Main Results}

The property of skew polynomial ring relation to Morita invariance, we have the following counterexample.

Example 3.1. Suppose that a ring $Z_{2}$ and $\sigma$ is an endomorphism over $Z_{2}$, define the usual addition and multiplication by $x a=\sigma(a) x$ for any $a \in \mathrm{Z}_{2}$, then $\mathrm{Z}_{2}[x ; \sigma]$ is a skew polynomial ring, but has no Morita invariance.

In fact, clearly, $\mathrm{Z}_{2}[x ; \sigma]$ is a skew polynomial ring. Consider a ring $\operatorname{Mat}_{2}\left(\mathrm{Z}_{2}[x ; \sigma]\right)=\left(\begin{array}{ll}f_{1}(x) & f_{2}(x) \\ f_{3}(x) & f_{4}(x)\end{array}\right)$, which $f_{1}(x)=\sum_{i=0}^{n} a_{i} x^{i}, \quad f_{2}(x)=\sum_{i=0}^{n} b_{i} x^{i}, \quad f_{3}(x)=\sum_{i=0}^{n} c_{i} x^{i}, \quad f_{4}(x)=\sum_{i=0}^{n} d_{i} x^{i} \in Z_{2}[x ; \sigma]$, we have all idempotents of $\operatorname{Mat}_{2}\left(\mathrm{Z}_{2}[x ; \sigma]\right)$ are $\left(\begin{array}{ll}1 & 0 \\ 0 & 1\end{array}\right),\left(\begin{array}{ll}0 & 0 \\ 0 & 0\end{array}\right),\left(\begin{array}{ll}0 & 0 \\ 0 & 1\end{array}\right),\left(\begin{array}{ll}1 & 0 \\ 0 & 0\end{array}\right),\left(\begin{array}{ll}1 & 1 \\ 0 & 0\end{array}\right),\left(\begin{array}{ll}1 & 0 \\ 1 & 0\end{array}\right)$. Suppose that $f_{i}(x) \neq 0, i=(1,2,3,4)$.

Case 1. If $e=\left(\begin{array}{ll}1 & 0 \\ 0 & 1\end{array}\right)$, then

$$
\begin{aligned}
\operatorname{Mat}_{2}\left(\mathrm{Z}_{2}[x ; \sigma]\right) e \operatorname{Mat}_{2}\left(\mathrm{Z}_{2}[x ; \sigma]\right) & =\left(\begin{array}{cc}
f_{1}^{2}(x)+f_{2}(x) f_{3}(x) & f_{1}(x) f_{2}(x)+f_{2}(x) f_{4}(x) \\
f_{3}(x) f_{1}(x)+f_{4}(x) f_{3}(x) & f_{3}(x) f_{2}(x)+f_{4}^{2}(x)
\end{array}\right) \\
& \neq\left(\begin{array}{cc}
f_{1}(x) & f_{2}(x) \\
f_{3}(x) & f_{4}(x)
\end{array}\right)=\operatorname{Mat}_{2}\left(\mathrm{Z}_{2}[x ; \sigma]\right)
\end{aligned}
$$

Case 2. If $e=\left(\begin{array}{ll}0 & 0 \\ 0 & 0\end{array}\right)$, then $\left(\begin{array}{ll}0 & 0 \\ 0 & 0\end{array}\right) \neq\left(\begin{array}{ll}f_{1}(x) & f_{2}(x) \\ f_{3}(x) & f_{4}(x)\end{array}\right)$. Case 3. If $e=\left(\begin{array}{ll}0 & 0 \\ 0 & 1\end{array}\right)$, then

$\left(\begin{array}{cc}f_{1}(x) & f_{2}(x) \\ f_{3}(x) & f_{4}(x)\end{array}\right) \neq\left(\begin{array}{cc}f_{2}(x) f_{3}(x) & f_{2}(x) f_{4}(x) \\ f_{4}(x) f_{3}(x) & f_{4}^{2}(x)\end{array}\right)$. Similarly, we show that

$\operatorname{Mat}_{2}\left(\mathrm{Z}_{2}[x ; \sigma]\right) e \operatorname{Mat}_{2}\left(\mathrm{Z}_{2}[x ; \sigma]\right) \neq \operatorname{Mat}_{2}\left(\mathrm{Z}_{2}[x ; \sigma]\right)$ for all idempotente. Clearly, the condition of

$\operatorname{Mat}_{n}\left(\mathrm{Z}_{2}[x ; \sigma]\right) e \operatorname{Mat}_{n}\left(\mathrm{Z}_{2}[x ; \sigma]\right)=\operatorname{Mat}_{n}\left(\mathrm{Z}_{2}[x ; \sigma]\right)$ is not true for any integer $\mathrm{n}$, so $\mathrm{Z}_{2}[x ; \sigma]$ and any ring $\mathrm{S}$ are not Morita equivalent by lemma 2.2, therefore $\mathrm{Z}_{2}[x ; \sigma]$ has no Morita invariance.

So the skew polynomial ring has no Morita invariance by the counterexample. The following theorem shows that the condition of skew polynomial ring constitutes Armendariz ring.

Theorem 3.2. Let $R$ be a ring that has no zero divisor and $\sigma$ be a monomorphism over $\mathrm{R}$, then skew polynomial ring $R[x ; \sigma]$ is an Armendariz ring.

Proof. Since $R$ has no zero divisor, so $R[x ; \sigma]$ has no zero divisor by lemma2.4, then $R[x ; \sigma]$ is a reversible ring. For any $f_{i}(x), g_{j}(x) \in R[x ; \sigma]$, and $l(x)=\sum_{j=0}^{m} g_{j}(x) y^{j}, h(x)=\sum_{i=0}^{n} f_{i}(x) y^{i} \in R[x ; \sigma][y]$, if

$$
\begin{aligned}
0 & =h(x) \cdot l(x)=\sum_{i+j=0}^{n+m} f_{i}(x) \sigma^{i}\left(g_{j}(x)\right) y^{i+j} \\
& =f_{0}(x) g_{0}(x)+\left(f_{0}(x) g_{1}(x)+f_{1}(x) g_{0}(x)\right) y+\cdots+f_{n}(x) g_{m}(x) y^{n+m}
\end{aligned}
$$

then the all coefficients of $R[x ; \sigma]$ are zero. Since $f_{i}(x) g_{j}(x)=0, f_{0}(x) g_{1}(x)+f_{1}(x) g_{0}(x)=0$, so $g_{1}(x) f_{0}(x)=0$, and hence $f_{0}(x) g_{1}(x)=0, f_{1}(x) g_{0}(x)=0$. Because

$f_{0}(x) g_{2}(x)+f_{1}(x) g_{1}(x)+f_{2}(x) g_{0}(x)=0, \quad f_{1}(x) g_{1}(x)+f_{2}(x) g_{0}(x)=0$, so $f_{1}(x) g_{1}(x)=0$,

$f_{2}(x) g_{0}(x)=0$, and have $g_{2}(x) f_{0}(x)=0, f_{0}(x) g_{2}(x)=0$. Similarly, $f_{i}(x) g_{j}(x)=0$

$(0 \leq i \leq n, 0 \leq j \leq m)$. Thus the skew polynomial ring $R[x ; \sigma]$ of no zero divisor is an Armendariz ring.

Next research the necessary and sufficient of this condition by the following example. 
Example 3.3. Let $R=\left(\begin{array}{cc}\mathrm{Z}_{2} & \mathrm{Z}_{2} \\ 0 & \mathrm{Z}_{2}\end{array}\right)$ be a ring with a monomorphism $\sigma$ defined by $\sigma\left(\left(\begin{array}{ll}a & b \\ 0 & c\end{array}\right)\right)=\left(\begin{array}{cc}a & b \\ 0 & -c\end{array}\right)$. For any $f(x)=\sum_{i=0}^{n} A_{i} x^{i}, g(x)=\sum_{j=0}^{m} B_{j} x^{j} \in R[x]$, define the usual addition and multiplication by $x A=\sigma(A) x$, then $R[x ; \sigma]$ is a skew polynomial ring, but $R[x ; \sigma]$ is not an Armendariz ring.

In fact, clearly, $R[x ; \sigma]$ is a skew polynomial ring. Let $f(x)=\left(\begin{array}{ll}1 & 0 \\ 0 & 0\end{array}\right)+\left(\begin{array}{ll}1 & 1 \\ 0 & 0\end{array}\right) x$,

$g\left(\begin{array}{ll}0 & 0 \\ 0 & 1\end{array}\right)+\left(\begin{array}{ll}0 & 1 \\ 0 & 1\end{array}\right) x \in R[x ; \sigma]$, if $f(x) \cdot g(x)=0$, then all coefficients of the skew polynomial $R[x ; \sigma]$ are zero. But $\left(\begin{array}{cc}1 & 0 \\ 0 & 0\end{array}\right) \cdot\left(\begin{array}{cc}0 & 1 \\ 0 & -1\end{array}\right)=\left(\begin{array}{cc}0 & 1 \\ 0 & 0\end{array}\right) \neq 0,\left(\begin{array}{cc}1 & 1 \\ 0 & 0\end{array}\right) \cdot\left(\begin{array}{cc}0 & 0 \\ 0 & -1\end{array}\right)=\left(\begin{array}{cc}0 & -1 \\ 0 & 0\end{array}\right) \neq 0$, thus $R[x ; \sigma]$ is not an Armendariz ring.

It derives from the above example 3.3 that we further verify the condition is necessary. Next we study that skew polynomial ring $R[x ; \sigma]$ is a (quasi)-Baer ring under the condition of no zero divisor .

Theorem 3.4. Let $R$ be a ring that has no zero divisor and $\sigma$ is an endomorphism over $R$, then skew polynomial ring $R[x ; \sigma]$ is a (quasi)-Baer ring.

Proof. For any $f(x)=\sum_{i=0}^{n} a_{i} x^{i}, g(x)=\sum_{j=0}^{m} b_{j} x^{j} \in R[x ; \sigma]$, let $f(x) \cdot g(x)=0$. If $f(x)=0$, then $g(x)$ is any polynomial ring, and has $r_{R[x ; \sigma]}(f(x))=R=1_{R[x ; \sigma]} \cdot R$. If $f(x) \neq 0$, there exists $a_{i} \neq 0$, such that $0=f(x) \cdot g(x)=\sum_{i+j=0}^{n+m} a_{i} x^{i} b_{j} x^{j}=\sum_{i+j=0}^{n+m} a_{i} \sigma^{i}\left(b_{j}\right) x^{i+j}$, then $a_{i} \sigma^{i}\left(b_{j}\right)=0$. And because $R$ is a ring has no zero divisor, so $a_{i}=0$ or $\sigma^{i}\left(b_{j}\right)=0$. Since arbitrary of $a_{i}$ and $i$, we have $\sigma^{i}\left(b_{j}\right)=0$ implies $b_{j}=0(0 \leq j \leq m)$, so $g(x)=0$. Thus the right annihilators set of any nonempty subset $X$ is $r_{R[x ; \sigma]}(X)=0=0 \cdot R$. So $R[x ; \sigma]$ is a Baer ring, and $R[x ; \sigma]$ is a quasi-Baer ring by [5].

The following example shows that skew polynomial ring is (quasi)-Baer ring.

Example 3.5. Let $R=Z_{2}=\{0,1\}$ be a ring with an endomorphism $\sigma$ defined by $\sigma(a)=-a$, then $R[x ; \sigma]$ is a skew polynomial ring, and $R[x ; \sigma]$ is a (quasi)-Baer ring.

In fact, clearly, $R$ is a field, so $R$ is a no zero divisor ring. Therefore the right annihilator of every nonempty subset $X \subset R$ is $r_{R[x ; \sigma]}(X)=0=0 \cdot R$, then the right ideal generated by the idempotent 0 . Thus $R[x ; \sigma]$ is a (quasi)-Baer ring clearly by theorem 3.4.

So we proof the condition of no zero divisor is necessary. The following counterexample shows that the condition is not sufficient condition that skew polynomial ring is a (quasi)-Baer ring.

Example 3.6. Suppose that $R=\left(\begin{array}{cc}\mathrm{Z}_{4} & \mathrm{Z}_{4} \\ 0 & \mathrm{Z}_{4}\end{array}\right)$ be a ring with an endomorphism $\sigma$ defined by $\sigma\left(\left(\begin{array}{ll}a & b \\ 0 & c\end{array}\right)\right)=\left(\begin{array}{cc}a & -b \\ 0 & c\end{array}\right)$ over $R$, for any $f(x)=\sum_{i=0}^{n} A_{i} x^{i}, g(x)=\sum_{j=0}^{m} B_{j} x^{j} \in R[x]$, define the usual addition and multiplication is defined by $x z=\sigma(z) x$, then $R[x ; \sigma]$ is a skew polynomial ring, but is not a (quasi)-Baer ring.

In fact, clearly, $R[x ; \sigma]$ is a skew polynomial ring. For any $f(x)=\sum_{i=0}^{n} A_{i} x^{i} \in R[x ; \sigma]$, if $f^{2}(x)=f(x)$, then $A_{0}^{2}=A_{0}, A_{0} A_{1}+A_{1} \sigma\left(A_{0}\right)=A_{1}, \cdots, A_{n} \sigma^{n}\left(A_{n}\right)=0$.We have all idempotents of $R[x ; \sigma]$ are $\left(\begin{array}{ll}1 & 0 \\ 0 & 1\end{array}\right)$, $\left(\begin{array}{ll}0 & 0 \\ 0 & 0\end{array}\right),\left(\begin{array}{ll}1 & 0 \\ 0 & 0\end{array}\right)+\left(\begin{array}{cc}0 & b_{1} \\ 0 & 0\end{array}\right) x+\cdots+\left(\begin{array}{cc}0 & b_{n} \\ 0 & 0\end{array}\right) x^{n},\left(\begin{array}{ll}0 & 0 \\ 0 & 1\end{array}\right)+\left(\begin{array}{cc}0 & b_{1} \\ 0 & 0\end{array}\right) x+\cdots+\left(\begin{array}{cc}0 & b_{n} \\ 0 & 0\end{array}\right) x^{n}$. Let $f(x)=\left(\begin{array}{ll}2 & 2 \\ 0 & 2\end{array}\right) \in R[x ; \sigma]$, since 


$$
r_{R[x ; \sigma]}(f(x))=\left\{\left(\begin{array}{cc}
a_{0} & b_{0} \\
0 & c_{0}
\end{array}\right)+\left(\begin{array}{cc}
a_{1} & b_{1} \\
0 & c_{1}
\end{array}\right) x+\cdots+\left(\begin{array}{cc}
a_{n} & b_{n} \\
0 & c_{n}
\end{array}\right) x^{n} \mid a_{i}, b_{i}, c_{i} \in \mathrm{Z}_{4}\right\} \neq e R[x]
$$

which $e^{2}=e$. Thus $R[x ; \sigma]$ is not a (quasi)-Baer ring.

\section{Conclusion}

Inthis paper, we show that skew polynomial ring has no Morita invariance by the counterexample, and give the condition that skew polynomial ring constitutes Armendariz and (quasi)-Baer ring, and verify that the condition is necessary, but not sufficient.

\section{Acknowledgements}

The authors thank the referee for very careful reading the manuscript and many valuable suggestions that improved the paper by much. This work was supported by the National Natural Science Foundation of China (11361063).

\section{References}

[1] Jin, L. (2014) Some Properties of Skew Polynomial Rings. MS Thesis, Yanbian University, Yanji.

[2] Rege, M.B. and Chhawchharia, S. (1997) Armendariz Rings. Proceedings of the Japan Academy, Series A, Mathematical Sciences, 73, 14-17. http://dx.doi.org/10.3792/pjaa.73.14

[3] Chan, Y.H., Nam, K. and Yang, L. (2010) Skew Polynomial Rings over Semiprime Rings. Journal of the Korean Mathematical Society, 47, 879-897.

[4] Birkenmeier, G.F., Kim, J.Y. and Park, J.K. (2001) Principally Quasi-Baer Rings. Communications in Algebra, 29, 122. http://dx.doi.org/10.1081/AGB-100001530

[5] Song, J.Q. (1997) Baer and Quasi-Baer Rings of Iterated Skew Polynomial Rings. Journal of Mathematical Research and Exposition, 26, 103-106.

[6] Lambek, J. (1996) Lectures on Modules and Rings. Blaisdell, Waltham.

[7] Jin, H.L. (2003) Principally Quasi-Baer Skew Group Ring and Fixed Rings. PhD Thesis, Pusan National University, Pusan.

[8] Cohn, P.M. (1999) Reversible Rings. Bulletin London Mathematical Society, 31, 641-648. http://dx.doi.org/10.1112/S0024609399006116

[9] Li, J. (2010) Condition for Morita Context Ring Become the (Quasi)-Baer Ring. Yanbian University, Yanji.

\section{Submit or recommend next manuscript to SCIRP and we will provide best service for you:}

Accepting pre-submission inquiries through Email, Facebook, Linkedin, Twitter, etc

A wide selection of journals (inclusive of 9 subjects, more than 200 journals)

Providing a 24-hour high-quality service

User-friendly online submission system

Fair and swift peer-review system

Efficient typesetting and proofreading procedure

Display of the result of downloads and visits, as well as the number of cited articles

Maximum dissemination of your research work

Submit your manuscript at: http://papersubmission.scirp.org/ 